\title{
Capsule ${ }^{13} \mathrm{C}$-urea breath test for the diagnosis of Helicobacter pyloriinfection
}

\author{
Nan-Jing Peng, Kwok-Hung Lai, Ren-Shyan Liu, Shui-Cheng Lee, Daw-Guey Tsay, Ching-Chu Lo, Huei-Hwa Tseng, \\ Wen-Keui Huang, Gin-Ho Lo, Ping-I Hsu
}

\begin{abstract}
Nan-Jing Peng, Daw-Guey Tsay, Department of Nuclear Medicine, Kaohsiung Veterans General Hospital, Kaohsiung, Taiwan, China Kwok-Hung Lai, Ching-Chu Lo, Gin-Ho Lo, Ping-I Hsu, Division of Gastroenterology, Department of Internal Medicine, Kaohsiung Veterans General Hospital, Kaohsiung, Taiwan, China

Huei-Hwa Tseng, Wen-Keui Huang, Department of Pathology and Laboratory Medicine, Kaohsiung Veterans General Hospital, Kaohsiung, Taiwan, China

Ren-Shyan Liu, Department of Nuclear Medicine, Taipei Veterans General Hospital and National Yang-Ming University, Taipei, Taiwan, China

Shui-Cheng Lee, Institute of Nuclear Energy Research, Taoyuan, Taiwan, China

Supported by Grant From the National Science Council, Taiwan NSC 90-2314-B-075B-008

Correspondence to: Dr. Ping-I Hsu, Division of Gastroenterology, Department of Internal Medicine, Kaohsiung Veterans General Hospital, 386, Ta-Chung 1st Road, Kaohsiung 813, Taiwan,

China. williamhsup@yahoo.com.tw

Telephone: +886-7-3422121-2075 Fax: +886-7-3461179

Received: 2004-07-17 Accepted: 2004-09-09
\end{abstract}

\begin{abstract}
AIM: To compare the accuracy of capsule ${ }^{13} \mathrm{C}$-urea breath test (UBT) with conventional invasive methods for the diagnosis of Helicobacter pylori infection.
\end{abstract}

METHODS: One hundred patients received CLO test, histological examination, culture and $100-$ or $50-\mathrm{mg}$ capsule UBT for the diagnosis of $\mathrm{H}$ pylori infection. $\mathrm{H}$ pylori infection was defined as those with positive culture or positive results from both histology and CLO test.

RESULTS: Both the sensitivity and specificity of the 100-mg capsule UBT $(n=50)$ were $100 \%$. The sensitivity and specificity of the 50-mg capsule UBT $(n=50)$ were 96.4 and $100 \%$, respectively. Taken together, the accuracy of capsule UBT $(n=100)$ was higher than that of CLO test, histology and culture $(100 \%$ vs $92 \%, 91 \%$ and $89 \%$, respectively; $P=0.035,0.018$ and 0.005 , respectively). Our data showed that the optimal timing of sampling for 100and 50-mg capsule UBT was 15-30 and 6-15 min, respectively.

CONCLUSION: Capsule UBT has a higher accuracy compared with biopsy-based tests. It is an ideal method for the diagnosis of $H$ pylori infection.

(C) 2005 The WJG Press and Elsevier Inc. All rights reserved.

Key words: ${ }^{13} \mathrm{C}$-urea breath test; Capsule; Helicobacter pylori, Oral urease
Peng NJ, Lai KH, Liu RS, Lee SC, Tsay DG, Lo CC, Tseng HH, Huang WK, Lo GH, Hsu PI. Capsule ${ }^{13} \mathrm{C}$-urea breath test for the diagnosis of Helicobacter pylori infection. World J Gastroenterol 2005; 11(9): 1361-1364

http://www.wjgnet.com/1007-9327/11/1361.asp

\section{INTRODUCTION}

${ }^{13} \mathrm{C}$-urea breath test (UBT) has become a highly reliable method for the non-invasive diagnosis of Helicobacter pylori $\left(H\right.$ pylori) infection ${ }^{[1-3]}$. It is the ideal test for those in whom endoscopy is not required because it offers the combination of simplicity, accuracy and absence of exposure to radioactivity ${ }^{[3-6]}$. It is also a good method in monitoring treatment success following anti- $H$ pylori therapy, because it can avoid the false negative result from the biopsy-based tests due to patchy distribution of bacteria in the gastric mucosa and the false positive result from serology caused by previous $H$ pylori infection.

The principle of UBT is based on the high urease activity of $H$ pylori in vivo. The orally administrated ${ }^{13} \mathrm{C}$-labeled urea is readily hydrolyzed by urease of the organism to ammonia and ${ }^{13} \mathrm{CO}_{2}$. The latter can be detected through the collection of the expired samples. However, it is important to note that some urease-containing oral floras are also capable of hydrolyzing urea ${ }^{[7,8]}$. The conventional UBT uses a liquid form of urea solution. On such way of urea ingestion, breath ${ }^{13} \mathrm{CO}_{2}$ may increase at an earlier sampling time after consumption due to the presence of oral urease ${ }^{[8]}$. Cleansing mouths and delayed sampling collection at least 10 min after consumption are commonly used to avoid false positive results.

To prevent the effect of oral urease activity, we have previously developed an endoscopic UBT for the diagnosis of $H$ pylori infection. This diagnostic method applied ${ }^{13} \mathrm{C}$ urea to the stomach through the working channel of endoscope, and thus could avoid contamination of urea from oral urease. Our data demonstrate that the new modality can rapidly discriminate between $H$ pylori-positive and $H$ pylori-negative patients at $2 \mathrm{~min}$ after the consumption of ${ }^{13} \mathrm{C}$-urea with $100 \%$ of both sensitivity and specificity. Additionally, its diagnostic accuracy is significantly higher than that of culture, histology and CLO test $t^{[9]}$. Nonetheless, endoscopic UBT is an invasive method for the diagnosis of $H$ pylori infection and it is not a good choice for the patients not requiring endoscopic examinations.

We therefore developed a capsule UBT to improve its clinical application. The patients swallowed urea-containing 
capsules during the test to prevent the release of urea before reaching the stomach. The aims of this study were to compare the accuracy of capsule UBT with conventional methods for the diagnosis of $H$ pylori infection and to find an optimal dosage and timing for sampling collection.

\section{MATERIALS AND METHODS \\ Patients}

From August 2001 to July 2003, 100 patients who attended the routine upper gastrointestinal endoscopy were included in the present study. Fifty-seven were males and 43 were females, aged 20-83 years. Criteria for exclusion included use of proton pump inhibitors within 1 mo before endoscopy, antibiotic ingestion within 1 mo before endoscopy, serious medical illness, and previous history of anti-H pylori therapy. Written informed consent was obtained from each subject. This study was approved by the Human Medical Research Committee of our hospital and by the Department of Health, Taiwan.

\section{Uppergastrointestinalendoscopy, CLOtest, histologyand culture}

During endoscopy, four gastric biopsy specimens were taken from lesser curvature $2 \mathrm{~cm}$ from the pylorus. Two specimens were fixed immediately in 10\% neutral-buffered formalin for histological examination, one for urease test (CLO test, Delta-West, Bently, Australia), and the other for culture. Samples were sent to different laboratories that were blind to the results of other tests. The CLO test was monitored for color change up to $24 \mathrm{~h}$ after the addition of the gastric tissue. The gel was not warmed above ambient temperature at any time during the incubation period. The specimens for microbiological examination were transferred with brain heart infusion in ice and inoculated onto the CDC anaerobic blood agar (Becton Dickinson Microbiology System, Cockeysville, Maryland). The agar was incubated at $35{ }^{\circ} \mathrm{C}$ for $2 \mathrm{~d}$ in a micro-aerophilic gas mixture containing $5 \% \mathrm{O}_{2}, 100 \mathrm{~mL} / \mathrm{L} \mathrm{CO}_{2}$, and $85 \% \mathrm{~N}_{2}$. Culture-positive patients were those with bacterial colonies grown in culture within $7 \mathrm{~d}$. The organisms were identified as $H$ pylori by Gram staining, colony morphology and positive oxidase, catalase and urease reaction. Histology-positive patients were those with curved organisms seen in H\&E-stained sections under microscope. Although there is no real gold standard for the diagnosis of $H$ pylori, cultures should be $100 \%$ specific if the procedures are performed properly. Use of culture alone as the gold standard may yield false negative results due to the inherent difficulties of culture. Hence, in the present study we defined patients with $H$ pylori infection as those with positive culture or positive results from histology and CLO test.

\section{Capsule UBT}

The capsule UBT was done within $7 \mathrm{~d}$ of the endoscopy before antibiotic treatment was given. The ${ }^{13} \mathrm{C}$-urea, 99atom $\%{ }^{13} \mathrm{C}$-labeled urea, was produced by the Institute of Nuclear Energy Research (INER), Taiwan. Of the 100 patients, 50 received $100-\mathrm{mg}$ capsule ${ }^{13} \mathrm{C}$-urea and 50 received 50-mg capsule ${ }^{13} \mathrm{C}$-urea. No test meal was used in this study. Patients were asked to fast for at least $6 \mathrm{~h}$. A baseline sample was collected in duplicate by breathing out through a straw into a $13 \mathrm{~mL}$ vacutainer tube. Patients then underwent a mouth washing by gargling to avoid the influence of oral urease, and received 100- or 50-mg capsule ${ }^{13} \mathrm{C}$-urea. Thereafter, patients were lying down and changing sides every $5 \mathrm{~min}$. Breath samples were collected from the subjects $2,4,6,8,10,15,20,25$ and $30 \mathrm{~min}$ after ${ }^{13} \mathrm{C}$-urea consumption. All samples were sent to INER where a continuous flow isotope ratio mass spectrometer (CF-IRMS) (Europa Scientific Ltd, Crewe, UK) was used for analysis. One of the authors performed the analysis without knowing the results of the status of $H$ pylori in the patients.

Values were expressed as an excess $\delta^{13} \mathrm{CO}_{2} \%$ excretion. The $\delta{ }^{13} \mathrm{CO}_{2}$ is the ratio of ${ }^{13} \mathrm{C}$ to ${ }^{12} \mathrm{C}$ in the sample compared to the Pee Dee Belemnite (PDB) standard. The equation is given as: $\delta{ }^{13} \mathrm{CO}_{2}=\left(R_{\text {samp }}-R_{\text {std }}\right) / R_{\text {std }} \times 1000 . R_{\text {samp }}$ and $R_{\text {std }}$ represent the ratios of ${ }^{13} \mathrm{C}$ to ${ }^{12} \mathrm{C}$ in sample and standard, respectively. Excess $\delta{ }^{13} \mathrm{CO}_{2}$ is the value of $\delta{ }^{13} \mathrm{CO}_{2}$ detected at various time point minus that at the baseline.

\section{Statistical analysis}

Sensitivity, specificity and accuracy of capsule UBT were calculated for various cutoff values at different time points according to the predefined gold standard. The optimal cutoff value was determined by comparing the positions of the full curve in a graph from the receiver operating characteristic (ROC) curves ${ }^{[10]}$. The optimal timing was defined as the time point at which the sums of the false negatives plus false positives were minimized. Student's $t$ test was used for statistical analysis at different time points of UBT values. $\chi^{2}$ test was used to determine the significance of differences between capsule UBT and biopsy-based modalities. Differences were considered statistically significant at $P<0.05$.

\section{RESULTS}

\section{Capsule UBT}

Table 1 shows the means and standard deviation of excess $\delta{ }^{13} \mathrm{CO}_{2}$ and $t$ test values of the 100 - and 50 -mg capsule UBT at different time points. In the 50 cases that received 100 -mg capsule ${ }^{13} \mathrm{C}$-urea, 18 were $H$ pylori-positive and 32 $H$ pylori-negative subjects. Breath ${ }^{13} \mathrm{CO}_{2}$ values were significantly higher in the $H$ pylori-positive subjects than those in the H pylori-negative subjects from 2 min. According to the ROC curves, the sensitivity and specificity were both $100 \%$ at $15 \mathrm{~min}$ with a cutoff value of $5 \delta^{13} \mathrm{CO}_{2} \%$, and at 20-30 min with a cutoff value of $4-5 \delta^{13} \mathrm{CO}_{2} \%$. The optimal sampling time for discriminating $H$ pylori-positive and $H$ pylori-negative patients was accordingly between 15 and 30 min with $100 \%$ sensitivity and $100 \%$ specificity. In the 50 cases who received 50 -mg capsule ${ }^{13} \mathrm{C}$-urea, 28 were $H$ pylori-positive and $22 \mathrm{H}$ pylori-negative subjects. Breath ${ }^{13} \mathrm{CO}_{2}$ values were significantly higher in the $H$ pylori-positive subjects than those in the $H$ pylori-negative subjects from 2 min (Table 1). The sum of the false negatives plus false positives was the smallest at 6-10 min with a cutoff value of $1 \delta{ }^{13} \mathrm{CO}_{2} \%$ and at $15 \mathrm{~min}$ with a cutoff value of $1-2 \delta$ ${ }^{13} \mathrm{CO}_{2} \%$. Therefore, the optimal timing was between 6 and 15 min with $96.4 \%$ sensitivity and $100 \%$ specificity. 
Table 1 Comparison of data from 100- and 50-mg capsule UBT at different time points

\begin{tabular}{|c|c|c|c|c|c|c|c|c|c|}
\hline Method & $2 \min$ & $4 \mathrm{~min}$ & $6 \mathrm{~min}$ & $8 \mathrm{~min}$ & $10 \mathrm{~min}$ & $15 \min$ & $20 \mathrm{~min}$ & $25 \mathrm{~min}$ & $30 \mathrm{~min}$ \\
\hline 100-mg H pylori (+) & $4.15(5.33)^{\mathrm{b}}$ & $9.8(11.02)^{\mathrm{b}}$ & $13.29(11.73)^{b}$ & $16.82(14.03)^{b}$ & $19.37(15.55)^{b}$ & $22.13(14.89)^{a, b}$ & $23.57(17.63)^{a, b}$ & $20.63(15.23)^{\mathrm{ab}}$ & $20.07(15.29)^{\mathrm{a}, \mathrm{b}}$ \\
\hline \multicolumn{10}{|l|}{ UBT } \\
\hline H pylori (-) & $0.39(1.08)$ & $0.32(0.58)$ & $0.45(1.66)$ & $0.29(1.32)$ & $0.48(1.42)$ & $0.31(1.05)$ & $0.42(1.08)$ & $0.35(0.87)$ & $0.53(0.9)$ \\
\hline 50-mg H pylori $(+)$ & $1.8(1.81)^{\mathrm{b}}$ & $6.63(5.39)^{\mathrm{b}}$ & $9.95(7.01)^{\mathrm{b}}$ & $11.68(7.48)^{\mathrm{b}}$ & $12.17(7.59)^{\mathrm{b}}$ & $12.79(7.29)^{\mathrm{b}}$ & $13.17(8)^{\mathrm{b}}$ & $12.56(8.02)^{b}$ & $11.62(6.75)^{b}$ \\
\hline \multicolumn{10}{|l|}{ UBT } \\
\hline H pylori (-) & $0.04(0.33)$ & $0.04(0.29)$ & $0.04(0.31)$ & $-0.01(0.33)$ & $0.01(0.31)$ & $-0.08(0.35)$ & $0.1(0.46)$ & $0.02(0.39)$ & $0.02(0.42)$ \\
\hline
\end{tabular}

Values are in excess $\delta{ }^{13} \mathrm{CO}_{2}$ excretion \% : mean \pm SD. ${ }^{a} P<0.05(100-\mathrm{mg}$ UBT H pylori $(+)$ vs 50 -mg UBT H pylori $(+))$, ${ }^{\mathrm{b}} \mathrm{P}<0.01($ H pylori $(+)$ vs $H$ pylori $(-))$.

\section{Comparison of capsule UBT and biopsy-based modalities}

Sensitivity, specificity and accuracy of all the diagnostic modalities used in the present study are shown in Tables 2-4. Compared with biopsy-based modalities, the accuracy of 100-mg capsule UBT was higher than that of the CLO test, histology and culture (Table 2, 100\% vs 96\%, 90 and $98 \%$ ), although the differences were not statistically significant $(P>0.05)$. The sensitivity and accuracy of 50-mg capsule UBT were significantly higher than those of culture (Table 3, 96.4\% vs 64.2\%, $P=0.005 ; 98 \%$ vs $80 \%, P=0.008$ ).

Table 2 Sensitivity, specificity and accuracy of tests for the diagnosis of $H$ pylori infection in patients with $100-\mathrm{mg}$ capsule UBT

\begin{tabular}{lccr}
\hline & Sensitivity (\%) & Specificity (\%) & Accuracy (\%) \\
\hline CLO test $(n=50)$ & $94.4(17 / 18)$ & $96.6(31 / 32)$ & $96(48 / 50)$ \\
Histology $(n=50)$ & $88.9(16 / 18)$ & $90.6(29 / 32)$ & $90(45 / 50)$ \\
Culture $(n=50)$ & $94.4(17 / 18)$ & $100(32 / 32)$ & $98(49 / 50)$ \\
100 -mg UBT $(n=50)$ & $100(18 / 18)$ & $100(32 / 32)$ & $100(50 / 50)$ \\
\hline
\end{tabular}

Table 3 Sensitivity, specificity and accuracy of tests for the diagnosis of $H$ pylori infection in patients with $50-\mathrm{mg}$ capsule UBT

\begin{tabular}{lccc}
\hline & Sensitivity (\%) & Specificity (\%) & Accuracy (\%) \\
\hline CLO test $(n=50)$ & $82.1(23 / 28)$ & $95.5(21 / 22)$ & $88(44 / 50)$ \\
Histology $(n=50)$ & $92.9(26 / 28)$ & $90.9(20 / 22)$ & $92(46 / 50)$ \\
Culture $(n=50)$ & $64.2(18 / 28)^{\mathrm{b}}$ & $100(22 / 22)$ & $80(40 / 50)^{\mathrm{b}}$ \\
50-mg UBT $(n=50)$ & $96.4(27 / 28)$ & $100(22 / 22)$ & $98(49 / 50)$ \\
\hline
\end{tabular}

${ }^{\mathrm{b}} P<0.01$ vs UBT.

Taken together (Table 4), the sensitivity of capsule UBT $(n=100)$ was significantly higher than that of culture $(97.8 \%$ vs $76.1 \%, P=0.004)$. It also had a higher specificity compared with histology (100\% vs $90.7 \%, P=0.057)$. Additionally, the accuracy of this new non-invasive modality was significantly higher than that of CLO test, histology and culture $(99 \%$ vs $92 \%, 91$ and $89 \%$, respectively; $P=0.035$, 0.018 and 0.005 , respectively).

Table 4 Sensitivity, specificity and accuracy of tests for the diagnosis of $H$ pylori infection in patients with 50 - or $100-\mathrm{mg}$ capsule UBT

\begin{tabular}{lccc}
\hline & Sensitivity (\%) & Specificity (\%) & Accuracy (\%) \\
\hline CLO test $(n=100)$ & $87(40 / 46)$ & $96.3(52 / 54)$ & $92(92 / 100)^{\mathrm{a}}$ \\
Histology $(n=100)$ & $91.3(42 / 46)$ & $90.7(49 / 54)^{1}$ & $91(91 / 100)^{\mathrm{a}}$ \\
Culture $(n=100)$ & $76.1(35 / 46)^{\mathrm{b}}$ & $100(54 / 54)$ & $89(89 / 100)^{\mathrm{b}}$ \\
UBT $(n=100)$ & $97.8(45 / 46)$ & $100(54 / 54)$ & $99(99 / 100)$
\end{tabular}

${ }^{\mathrm{a}} P<0.05,{ }^{\mathrm{b}} P<0.01,{ }^{1} P=0.057$ vs UBT.

\section{DISCUSSION}

Since ${ }^{13} \mathrm{C}$-UBT was first described by Graham et a ${ }^{[11]}$, attention has been drawn to its methodology. The protocol has been modified by several investigators to make its use easier and to improve its accuracy. According to our previous studies, the false-positive rates of the conventional UBT still range from 4.3 to $10 . \%^{[6,8]}$. In the present study, we demonstrated that capsule UBT was an extremely accurate method for the diagnosis of H pylori infection. The diagnostic accuracy of this new modality was significantly higher than that of CLO test, histology and culture. The sensitivity and specificity of capsule UBT were 97.8 and $100 \%$, respectively. There were no false-positive cases because capsule UBT effectively avoided the contamination of urea from oral urease. Therefore, capsule UBT is probably an ideal method to diagnose $H$ pylori infection.

In the present study, although breath ${ }^{13} \mathrm{CO}_{2}$ values of the $H$ pylori-positive subjects were significantly greater than those of the $H$ pylori-negative subjects at $2 \mathrm{~min}$ and later (Table 1), the optimal timing of capsule UBT for a good sensitivity and specificity was still at $6 \mathrm{~min}$ and later. This might be because the mean time between intake and complete capsule disintegration in the stomach is $3 \mathrm{~min}(1.5-4 \mathrm{~min})^{[12]}$. Furthermore, the swallowed saliva with oral urease activity may contribute to exhaled ${ }^{13} \mathrm{CO}_{2}$ as late as $30 \mathrm{~min}$ after ${ }^{13} \mathrm{C}$ urea consumption ${ }^{[8,12]}$. Therefore, according to the present study, the optimal sampling time for discriminating H pyloripositive and $H$ pylori-negative patients is between 15 and $30 \mathrm{~min}$ on 100-mg capsule UBT and between 6 and $15 \mathrm{~min}$ on 50-mg capsule UBT.

In our study with $50-\mathrm{mg}$ capsule UBT, breath ${ }^{13} \mathrm{CO}_{2}$ values were significantly higher in the $H$ pylori-positive subjects than those in the $H$ pylori-negative subjects from 2 min. The optimal timing for $96.4 \%$ sensitivity and $100 \%$ specificity was between 6 and $15 \mathrm{~min}$. The efficacy of 50-mg capsule UBT to discriminate $H$ pylori-positive group from $H$ pylori-negative group was less than that with $100-\mathrm{mg}$ capsule UBT (Table 1). The breath ${ }^{13} \mathrm{CO}_{2}$ values were significantly lower in the $H$ pylori-positive subjects of 50-mg capsule UBT than in those of $100-\mathrm{mg}$ capsule UBT from 15 to $30 \mathrm{~min}(P<0.05)$. Several studies have suggested that the levels of H pylori too low to be detected by histology are usually associated with a negative UBT ${ }^{[13,14]}$. Furthermore, the urease concentrations in different $H$ pylori strains vary by up to eight-fold ${ }^{[15,16]}$. Therefore, the less sensitive result might be due to the lower dosage of ${ }^{13} \mathrm{C}$-urea in a weakly urease-positive bacterial strain or the low bacterial loads in the stomach of some infected patients. Capsule UBT 
with $50 \mathrm{mg}{ }^{13} \mathrm{C}$-urea may reduce the cost of the agent and maintain the specificity, but halving the dosage-reduced sensitivity of test.

Theoretically, endoscopic UBT is the most accurate method for the diagnosis of $H$ pylori infection since it avoids the influence of the urease activity in the oral cavity and can prevent the sampling bias by biopsy for histological examinations. In the previous study, we found that endoscopic UBT examination is a rapid and accurate method for the diagnosis of $H$ pylori infection, superior to the conventional UBT ${ }^{[9]}$. Suto et a ${ }^{[17]}$, have found positive correlations between the endoscopic UBT values and the $H$ pylori colonization and activity score in the antrum and corpus, and negative correlations between the endoscopic UBT values and the atrophy and intestinal metaplasia scores in the corpus. Therefore, the values of test results are also able to reflect the bacterial load in the stomach. However, endoscopy is invasive and expensive, causes the patient discomfort, and introduces the risk of endoscopy crossinfection. Application of encapsulated urea has the same preventive effect of endoscopic UBT against the release of urea before reaching the stomach and the contamination of oral urease.

Biopsy-based tests have the possibility of sampling error and may cause false negatives. UBT effectively integrates the enzyme activity of bacteria over the entire surface of the stomach, and avoids the possibility of false-negative results. In this study, we demonstrated that the capsule UBT was a rapid and extremely accurate method for the diagnosis of Hpylori infection. The diagnostic accuracy was significantly higher than that of CLO test, histology and culture.

In conclusion, our study shows that capsule UBT is a simple, noninvasive, rapid and extremely accurate method for the diagnosis of $H$ pylori infection. It has a higher accuracy compared with biopsy-based tests and is probably the choice of method for the diagnosis of $H$ pylori infection. It merits further studies to compare capsule UBT with conventional UBT and stool antigen test.

\section{ACKNOWLEDGEMENTS}

We thank Luo-Ping Ger, MPH, for statistical assistance and Miss Li-Chun Lin for her assistance in the procedure of capsule UBT and data management.

\section{REFERENCES}

1 Cutler AF, Havstad S, Ma CK, Blaser MJ, Perez-Perez GI, Schubert TT. Accuracy of invasive and noninvasive tests to diagnose Helicobacter pylori infection. Gastroenterology 1995; 109: 136-141

2 Goddard AF, Logan RP. Review article: urea breath tests for detecting Helicobacter pylori. Aliment Pharmacol Ther 1997; 11:
641-649

3 Slomianski A, Schubert T, Cutler AF. $\left[{ }^{13} \mathrm{C}\right]$ urea breath test to confirm eradication of Helicobacter pylori. Am J Gastroenterol 1995; 90: 224-226

4 Epple HJ, Kirstein FW, Bojarski C, Frege J, Fromm M, Riecken EO, Schulzke JD. ${ }^{13} \mathrm{C}$-urea breath test in Helicobacter pylori diagnosis and eradication. Correlation to histology, origin of 'false' results, and influence of food intake. Scand J Gastroenterol 1997; 32: 308-314

5 Katelaris $\mathbf{P H}$, Jones DB. Testing for Helicobacter pylori infection after antibiotic treatment. Am J Gastroenterol 1997; 92: 1245-1247

6 Peng NJ, Hsu PI, Lee SC, Tseng HH, Huang WK, Tsay DG, Ger LP, Lo GH, Lin CK, Tsai CC, Lai KH. A 15-minute $\left[{ }^{13} \mathrm{C}\right]-$ urea breath test for the diagnosis of Helicobacter pylori infection in patients with non-ulcer dyspepsia. J Gastroenterol Hepatol 2000; 15: 284-289

7 Peng NJ, Hsu PI, Lee SC, Tseng HH, Huang WK, Tsay DG, Lo GH, Lin CK, Cheng JS, Lai KH. Follow-up of Helicobacter pylori status by using ${ }^{13} \mathrm{C}$-urea breath test in nonulcer dyspeptic patients after eradication therapy. Zhonghua Yixue Zazhi (Taipei) 2001; 64: 337-342

8 Peng NJ, Lai KH, Liu RS, Lee SC, Tsay DG, Lo CC, Tseng $\mathrm{HH}$, Huang WK, Lo GH, Hsu PI. Clinical significance of oral urease in diagnosis of Helicobacter pylori infection by [13C] urea breath test. Dig Dis Sci 2001; 46: 1772-1778

9 Peng NJ, Lai KH, Liu RS, Lee SC, Tsay DG, Lo CC, Tseng $\mathrm{HH}$, Huang WK, Lo GH, Hsu PI. Endoscopic ${ }^{13} \mathrm{C}$-urea breath test for the diagnosis of Helicobacter pylori infection. Dig Liver Dis 2003; 35: 73-77

10 Zweig MH, Campbell G. Receiver-operating characteristic (ROC) plots: a fundamental evaluation tool in clinical medicine. Clin Chem 1993; 39: 561-577

11 Graham DY, Klein PD, Evans DJ, Evans DG, Alpert LC, Opekun AR, Boutton TW. Campylobacter pylori detected noninvasively by the ${ }^{13} \mathrm{C}$-urea breath test. Lancet $1987 ; \mathbf{1}$ : 1174-1177

12 Hamlet AK, Erlandsson KI, Olbe L, Svennerholm AM, Backman VE, Pettersson AB. A simple, rapid, and highly reliable capsule-based ${ }^{14} \mathrm{C}$ urea breath test for diagnosis of Helicobacter pylori infection. Scand J Gastroenterol 1995; 30: 1058-1063

13 Epple HJ, Kirstein FW, Bojarski C, Frege J, Fromm M, Riecken EO, Schulzke JD. ${ }^{13} \mathrm{C}$-urea breath test in Helicobacter pylori diagnosis and eradication. Correlation to histology, origin of 'false' results, and influence of food intake. Scand J Gastroenterol 1997; 32: 308-314

14 Graham DY, Klein PD. Accurate diagnosis of Helicobacter pylori. ${ }^{13} \mathrm{C}$-urea breath test. Gastroenterol Clin North Am 2000; 29: 885-893, $x$

15 Ferrero RL, Hazell SL, Lee A. The urease enzymes of Campylobacter pylori and a related bacterium. J Med Microbiol 1988; 27: 33-40

16 Perri F, Clemente R, Pastore M, Quitadamo M, Festa V, Bisceglia M, Li Bergoli M, Lauriola G, Leandro G, Ghoos Y, Rutgeerts $\mathrm{P}$, Andriulli A. The ${ }^{13} \mathrm{C}$-urea breath test as a predictor of intragastric bacterial load and severity of Helicobacter pylori gastritis. Scand J Clin Lab Invest 1998; 58: 19-27

17 Suto H, Azuma T, Ito S, Ito Y, Miyaji H, Yamazaki Y, Kohli $\mathrm{Y}$, Kuriyama M. Endoscopic $\left[{ }^{13} \mathrm{C}\right]$-urea breath test for quantification of Helicobacter pylori infection. J Gastroenterol Hepatol 2000; 15: 161-167

Assistant Editor Guo SY Edited by Wang XL and Gabbe M 